\title{
Recherche chronologique sur la culture mochica du Pérou : datation de la tombe du Prêtre de Sipán par thermoluminescence (TL) et par radiocarbone
}

Céline Roque, Emmanuel Vartanian, Pierre Guibert, Max Schvoerer, Daniel Lévine, Walter Alva et Hogne Jungner

\section{(2) OpenEdition \\ Journals}

Édition électronique

URL : http://journals.openedition.org/jsa/2776

DOI : 10.4000/jsa.2776

ISSN : $1957-7842$

Éditeur

Société des américanistes

Édition imprimée

Date de publication : 1 janvier 2002

Pagination : 227-243

ISSN : 0037-9174

\section{Référence électronique}

Céline Roque, Emmanuel Vartanian, Pierre Guibert, Max Schvoerer, Daniel Lévine, Walter Alva et Hogne Jungner, « Recherche chronologique sur la culture mochica du Pérou : datation de la tombe du Prêtre de Sipán par thermoluminescence (TL) et par radiocarbone », Journal de la société des américanistes [En ligne], 88 | 2002, mis en ligne le 05 janvier 2007, consulté le 30 avril 2019. URL http://journals.openedition.org/jsa/2776 ; DOI : 10.4000/jsa.2776 


\title{
NOTE DE RECHERCHE
}

\author{
RECHERCHE CHRONOLOGIQUE \\ SUR LA CULTURE MOCHICA DU PÉROU : \\ DATATION DE LA TOMBE DU PRÊTRE DE SIPÁN \\ PAR THERMOLUMINESCENCE (TL) ET PAR RADIOCARBONE
}

\author{
C. ROQUE *, E. VARTANIAN *, P. GUIBERT *, \\ M. SCHVOERER *, D. LÉVINE **, W. ALVA ***, H. JUNGNER ****
}

La Culture mochica du Pérou et le site de SipÁn : PROBLÉMATIQUE CHRONOLOGIQUE

De l'importance des recherches chronologiques sur la culture mochica du Pérou

La culture Moche, ou mochica, est sans doute l'une des plus brillantes de l'époque pré-Inca. Elle s'épanouit sur la côte nord du Pérou, parallèlement aux civilisations Paracas Nécropolis et Nasca, qui se développent sur la côte sud du pays. Elle marque l'apogée d'un art particulièrement rafliné qui se manifeste notamment à travers l'orfèvrerie et la céramique, dont l'iconographie, très riche, apporte des témoignages irremplaçables sur cette société et ses mythes (Alcina Franch 1996; Bawden 1996 ; Curatola y Silva-Santisteban 1995; Watanabe 1995).

Une remise en cause récente des connaissances

Les découvertes archéologiques récentes ont conduit à une réévaluation complète des connaissances acquises anciennement sur les Mochicas. Le premier colloque international portant sur cette culture, organisé en 1994 à Trujillo, a été l'occasion,

*Institut de recherche sur les archéomatériaux, UMR5060 du CNRS, Centre de recherche en physique appliquée à l'archéologie (CRPAA), Université Bordeaux 3, Maison de l'archéologie, 33607 Pessac cedex, France. E-mail : guibert@montaigne.u-bordeaux.fr

** Université Paris-Sorbonne (Paris IV), UFR Art et archéologie, 3 rue Michelet, 75006 Paris, France.

*** Instituto nacional de cultura, Museo arqueológico nacional Brüning, Av. Huamachuco s/n, Apartado 33, Lambayeque, Perú.

**** Dating Laboratory, University of Helsinki, POB 11, 00014 Helsinki, Finlande. E-mail : hogne.jungner@helsinki.fi

Journal de la Société des Américanistes, 2002, 88 : p. 227 à 243. Copyright (C) Société des Américanistes. 
pour les archéologues, de remettre en question les interprétations traditionnelles, qu'elles soient chronologiques, stylistiques ou iconographiques, fondées sur l'étude de la céramique (Uceda y Mujica 1994). Il apparaît ainsi qu'un des problèmes cruciaux rencontrés dans l'étude de la culture mochica est celui de la chronologie. De véritables divergences apparaissent quant à son calage dans le temps, ses limites chronologiques étant placées de manière très floue entre le ${ }^{\mathrm{e}}{ }^{\mathrm{e}}$ siècle av. $\mathrm{J}-\mathrm{C}$ et le $\mathrm{VII}^{\mathrm{e}}$ siècle apr. J.-C. (Makowski Hanula, 1995 ; Lévine 2001).

\section{Une chronologie à bâtir}

C’est la sériation morphologique et stylistique des céramiques, développée par Larco Hoyle (1948), affinée par Donnan $(1965,1976)$ puis Donnan et Mc Clelland (1979), qui a été le plus largement utilisée afin d'établir la chronologie de la culture mochica. Cependant, les avancées de l'archéologie péruvienne et l'accroissement progressif du cortège des datations physiques montrent les limites de pertinence de cette séquence, qui manque cruellement de confirmations stratigraphiques et chronologiques (Canziani et al. 1994 ; Arsenault 1995 ; Bawden 1996). Larco Hoyle présupposait en effet que les variations stylistiques observées correspondaient à différentes étapes du développement temporel de cette culture. Or elles ne se produisent pas de la même manière dans toutes les zones géographiques et ne sont pas nécessairement synchrones (Canziani et al. 1994) ; elles peuvent également refléter des traditions locales diverses. La mise en évidence d'une dichotomie entre le nord et le sud du territoire (Uceda y Mujica 1994 ; Watanabe 1995) permet de penser que la succession chronologique des formes resterait applicable dans les vallées de Chicama et de Moche, à partir desquelles elle a été développée, mais il ne semble pas possible a priori de l'étendre à d'autres régions (Canziani et al. 1994). Cette typo-chronologie perd donc son caractère universel : son cadre d'applicabilité n'est pas clair, notamment pour la production céramique issue des vallées septentrionales du Pérou.

Finalement, les découvertes récentes de tombes mochicas au nord du territoire, dont celles de Sipán, appellent des réserves sur l'extension de la typo-chronologie de Larco Hoyle à la totalité de l'aire géographique occupée par les Mochicas entre le $\mathrm{II}^{\mathrm{e}}$ siècle av. J.-C. et le VIII ${ }^{\mathrm{e}}$ siècle apr. J.-C. Il apparaît donc nécessaire de l'affiner grâce à des datations physiques plus systématiques, à condition de disposer de données archéologiques scientifiquement rigoureuses (Shimada 1994 ; Lévine 2001 ; Uceda 2000). De tels impératifs sont satisfaits dans la nécropole princière de Sipán dont la datation, par croisement de méthodes, doit permettre de contribuer de manière pertinente à la résolution de cette problématique.

\section{La nécropole princière de Sipán}

Le site archéologique de Sipán, appelé Huaca Rajada, est localisé sur la côte nord du Pérou, dans le département de Lambayeque, l'une des régions les mieux irriguées et les plus prospères de l'Ancien Pérou. Sa richesse en ressources minières explique pourquoi c'est de ce territoire que proviennent les découvertes les plus abondantes et les plus importantes en matière d'orfèvrerie et de métallurgie pré-hispaniques, issues des tombes des élites gouvernantes (Collin Delavaud 1984; Alva 1994). 


\section{Description du complexe}

Le complexe funéraire de Sipán se caractérise par la présence de deux grandes structures pyramidales d'adobe, aujourd'hui érodées, qui émergent entre les plantations actuelles de canne à sucre. Il s'agit d'édifices massifs, alignés selon un axe ouest-est, séparés par une place d'environ $60 \mathrm{~m}$ de côté et au pied desquels une plate-forme funéraire a été mise au jour. Occupant une aire de $130 \mathrm{~m}$ sur $50 \mathrm{~m}$, elle se compose de trois niveaux distincts, dont le premier s'élève à environ $8 \mathrm{~m}$ au-dessus de la plaine. De nombreuses sépultures y ont été identifiées et fouillées ; les plus importantes sont celles du «Seigneur de Sipán », du «Vieux Seigneur de Sipán », du « Prêtre », du « Guerrier » et du « Jeune » (Figure 1).

\section{Intérêt archéologique}

La Huaca Rajada de Sipán apparaît aujourd'hui comme un important centre cérémoniel attribué à la culture mochica. Les fouilles, menées de manière exhaustive et scientifique, ont apporté des informations nouvelles sur cette société. Il s'agit là d'un travail sans précédent dans l'archéologie péruvienne, qui ouvre de nouvelles perspectives pour l'interprétation de cette culture (Alva \& Donnan 1993 ; Alva 1994). Ainsi, l'étude de l'architecture du site, de l'organisation et du contenu des tombes fouillées permet de considérer cette plate-forme comme une sorte de "mausolée royal » destiné, pour quelques générations, à l'inhumation des membres de l'élite gouvernante locale et de son entourage (Alva 1994). Cette découverte permet d'étayer la thèse de l'organisation du territoire mochica, depuis la région de Piura au Nord jusqu'à Huarmey au Sud, en seigneuries autonomes, l'une d'elles ayant pour centre le site de Sipán (Bawden 1994 ; Shimada 1994).

\section{Données chronologiques}

À partir de l'étude de l'architecture du complexe, de la typologie des constructions et du matériel retrouvé dans les tombes (en particulier la céramique), les archéologues ont estimé que le site aurait été principalement occupé entre le $\mathrm{I}^{\mathrm{er}}$ et le $\mathrm{IV}^{\mathrm{e}}$ siècle apr. J.-C. (Alva 1988 ; Alcina Franch 1996). Six phases de construction, probablement associées à une évolution du rituel, ont été identifiées dans la plate-forme funéraire et permettent de dégager une chronologie relative entre les sépultures (Alva 1990 et 1994 ; Schuster 1992). Ainsi, la tombe du «Vieux Seigneur », attribuée aux phases Moche I et II, serait la plus ancienne ; celles du « Prêtre » et du " Seigneur » appartiendraient à la période Moche III et au début de Moche IV. Par ailleurs, il est important de remarquer que les céramiques retrouvées en abondance dans les sépultures ne portent pas, à quelques rares exceptions, d'anse en étrier, élément habituellement considéré comme caractéristique des vases mochicas, et sur lequel repose la typo-chronologie de R. Larco Hoyle. L'utilisation de ce trait comme marqueur chronologique est donc impossible à Sipán.

Malgré l'importance du complexe funéraire de Sipán pour la connaissance archéologique et chronologique de la culture mochica, une seule datation physique était connue pour le site au début de nos travaux : il s'agit d'une date radiocarbone effectuée sur un fragment de bois issu de la tombe du « Seigneur » (Beta 23147, Beta Analytic Inc, Radiocarbon Dating Services, Miami). Le résultat obtenu, $260 \pm 90$ AD (Alva 


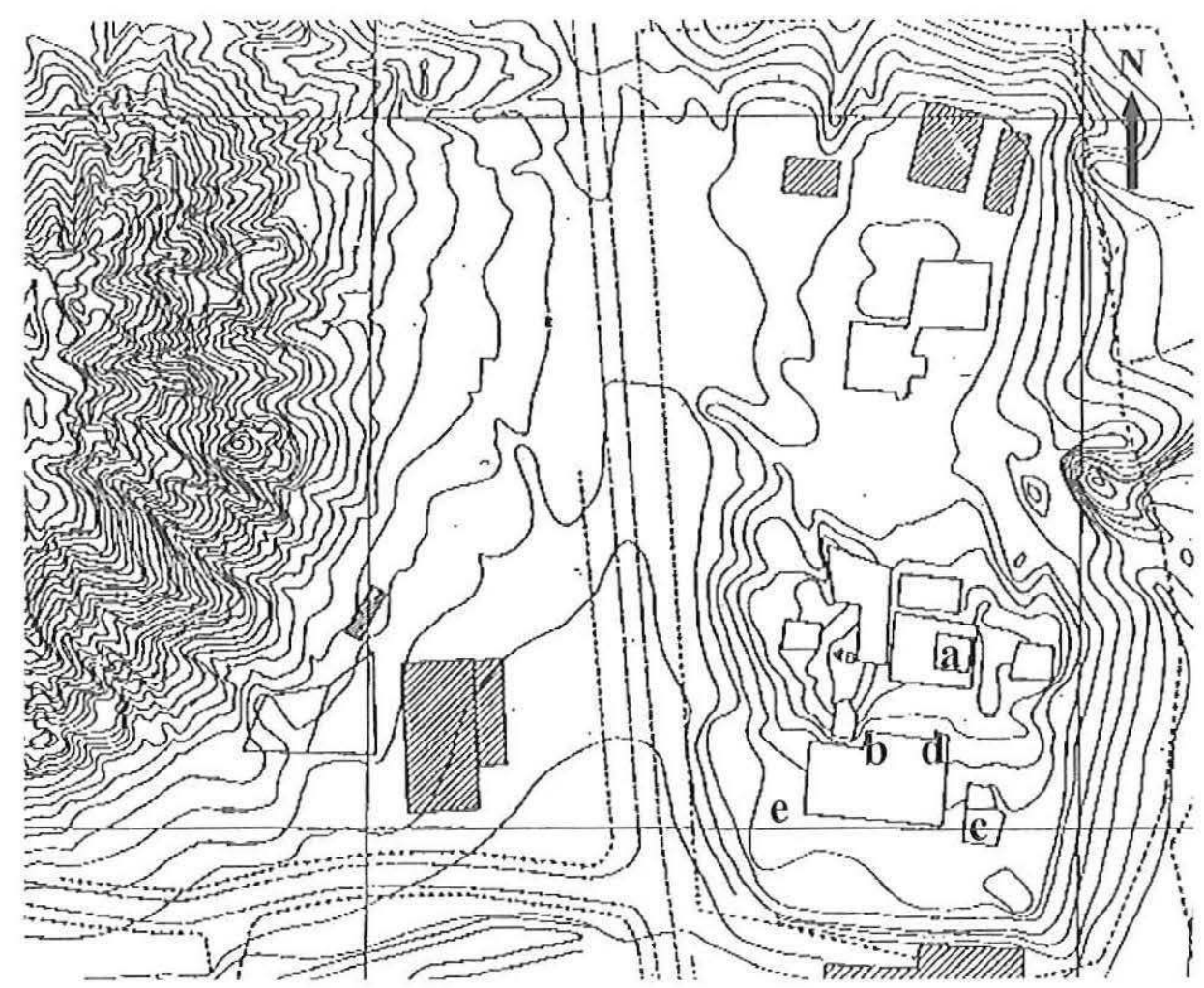

FIG. 1 - Plan du site archéologique de Sipán (d'après Alva 1994).

Il se compose de deux pyramides au pied desquelles se développe la zone des sépultures. On y trouve la tombe du Seigneur (a), celle du Vieux Seigneur (b), celle du Prêtre (c), celle du Guerrier (d), celle du Jeune (e).

1988), n'est pas présenté de manière conventionnelle, et il n'est pas possible de savoir s'il est calibré ou non. Cette donnée chronologique apparaît donc aujourd'hui inexploitable.

Enfin, la nécropole princière de Sipán n'a fait l'objet d'aucune campagne systématique de datations permettant d'établir sa chronologie de manière fiable et précise. C'est pour tenter de combler ce sérieux manque qu'un vaste programme de recherche à caractère chronologique a été engagé sur le site, au titre de la coopération PACTEurope/PACT-Amérique Latine. La présence d'un abondant matériel céramique permettait d'envisager une série de datations par thermoluminescence, sur des échantillons provenant des tombes du Prêtre, du Guerrier, du Jeune et du Vieux Seigneur. En parallèle, une datation par le radiocarbone a été effectuée pour la tombe du Prêtre, dont l'étude chronologique est présentée ici. 


\section{La tombe du Prêtre : le matériel étudié (Figure 2)}

Cette sépulture a été mise au jour en 1991, quelques années après celle du Seigneur de Sipán (Alva 1994; Donnan \& Castillo 1992). Placée à l'extrémité sud-est de la plate-forme funéraire et creusée à environ $4 \mathrm{~m}$ de profondeur, elle est de plan carré et ses côtés, d'une longueur de $4 \mathrm{~m}$, sont orientés selon les points cardinaux. Au moment de sa découverte, la chambre funéraire était totalement comblée par un remplissage homogène fait de terre granuleuse et de fragments d'adobe, détachés des parois. Le Prêtre a été inhumé avec un cortège d'accompagnants, sacrifiés, composé d'hommes, de femmes, d'un enfant et d'animaux (chien, serpent, lama). Dans les murs de la chambre funéraire parementés de briques d'adobe, sont ménagées des niches à offrandes ou hornacinas, contenant des vases en terre cuite à la fonction votive et symbolique.

L'organisation et le contenu des tombes du Prêtre et du Seigneur sont similaires. De plus, la proximité des deux enceintes funéraires et leur architecture sont autant d'indices qui ont amené les archéologues à envisager leur contemporanéité (Alva 1994).

La datation par thermoluminescence (TL) a été menée sur des tessons provenant de cinq céramiques différentes, recueillis dans les quatre hornacinas identifiées. Afin de mener à bien la procédure, des échantillons de sédiment ont été prélevés dans l'environnement immédiat des tessons à dater (Tableau 1 , infira) et des mesures de radioactivité ont été réalisées au moment de l'échantillonnage. L'observation de l'organisation de la tombe, et en particulier des parois, ne révèle pas de traces de percements secondaires qui auraient pu indiquer une réouverture de la cavité et un remaniement de son contenu après le scellement initial. Les céramiques ont par conséquent été déposées au moment de l'inhumation du Prêtre et leur datation par thermoluminescence (TL) permet donc de dater la sépulture. La Figure 2 présente la sépulture du Prêtre en fin de fouille (la dépouille du Prêtre a été déposée) et montre les différents éléments du matériel recueilli dans leur contexte archéologique.

Par ailleurs, une datation par radiocarbone a été réalisée au laboratoire d'Helsinki (Finlande) sur un fragment de poutre en bois provenant du plafond scellant la chambre funéraire (BDX 5138).

\section{ÉLÉMENTS SUCCINCTS SUR LA MÉTHODE DE DATATION PAR THERMOLUMINESCENCE}

Il n'est pas question de refaire ici un exposé détaillé du principe de la méthode, présenté par ailleurs (Aitken 1985). Il sera rappelé simplement que l'obtention d'un âge passe in fine par la détermination de deux grandeurs appelées dose d'irradiation archéologique (ou dose naturelle) et dose d'irradiation annuelle. L'âge est obtenu par le rapport de l'une à l'autre :

Âge TL $=$ Dose archéologique $/$ Dose annuelle $=\frac{\text { Qnat }}{\mathrm{I}}$ 


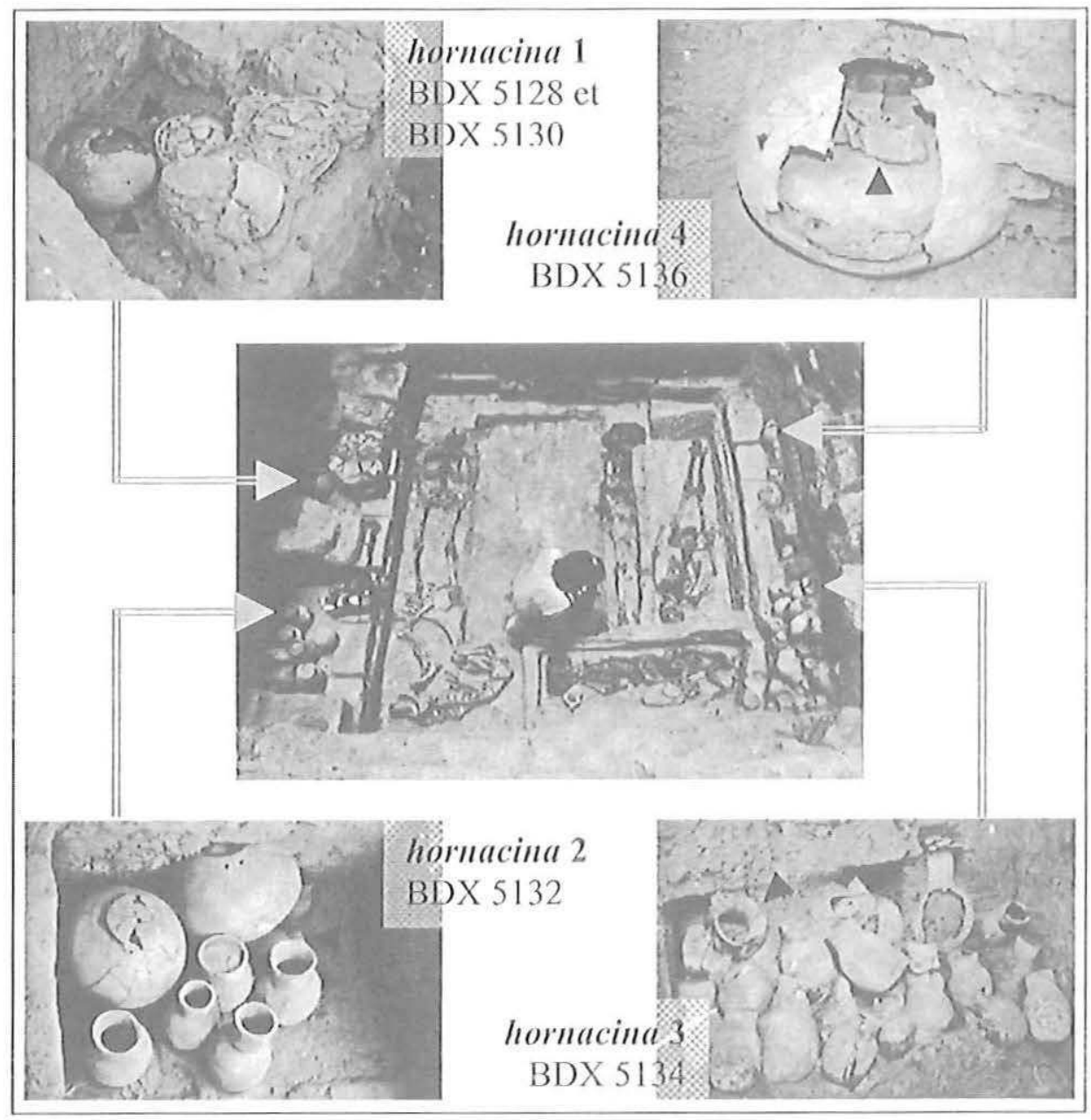

Fig. 2 - Sipán : La tombe du Prêtre en fin de fouille (Alva 1994). Autour de la sépulture centrale (le squelette du Prêtre a été déposé), on trouve les " accompagnants » : deux femmes, un homme placé dans un cercueil en bambou, un enfant accompagné d'un chien, les restes d'un serpent et un lama décapité. Le matériel recueilli provient des hornacinas (dépôts d'oflirande), ménagées dans les parois latérales est et ouest de la tombe.

\section{Détermination des doses d'irradiation archéologiques Qnat $\beta$ et Qnata}

Les échantillons à dater ont été préparés selon la technique des petites inclusions, dont le protocole est décrit par ailleurs (Schvoerer et al. 1994 ; Guibert et al. 1998). Dans le cas présent, étant donnée la faible épaisseur des céramiques (4 à $7 \mathrm{~mm}$ ), il a été choisi d'éliminer seulement $1 \mathrm{~mm}$ sur chaque face des échantillons au lieu des $2 \mathrm{~mm}$ requis. Il sera tenu compte dans la détermination de la dose annuelle de la fraction de dose $\beta$ provenant du sol (Aitken et al. 1985). Par ailleurs, en plus des traitements chimiques usuels, une attaque à l'acide fluorhydrique dilué ( $2 \%$ pendant 45 minutes) 
a été effectuée afin d'éliminer une grande partie de la fraction argileuse frittée aux grains luminescents. Ce décapage a pour effet, en particulier, d'accroître l'intensité de la thermoluminescence des cristaux, souvent faible pour les échantillons jeunes.

La dose d'irradiation accumulée par les céramiques depuis leur dernier chauffage a été mesurée par thermoluminescence selon une procédure d'ajout de dose suivie d'une régénération. Elle a nécessité l'utilisation de deux lots distincts de prises d'essai contenant les cristaux irradiés naturellement. L'un permet d'obtenir une première série d'enregistrements dite de « $1^{\text {re }}$ lecture TL » effectuée au cours du premier chauffage du matériau au laboratoire : des doses d'irradiation artificielles sont ajoutées à l'irradiation naturelle grâce à des sources radioactives calibrées, $\beta$ ou $\alpha$ selon le cas. L'autre lot a été soumis à un chauffage à $400^{\circ} \mathrm{C}$ dans l'air pendant 16 heures destiné à éliminer la thermoluminescence naturelle des cristaux tout en respectant leurs propriétés de luminescence originelles. Puis, après avoir subi des irradiations au laboratoire, ces prises d'essai permettent d'acquérir une seconde série d'enregistrements dite de « $2^{\mathrm{e}}$ lecture TL » qui conduit à déterminer la loi d'acquisition de la TL en fonction de la dose d'irradiation. Cette « courbe d'étalonnage » spécifique aux cristaux à dater, approchée par une fonction polynomiale (Guibert et al. 1996), peut alors être transposée sur les points expérimentaux de « $1^{\text {re }}$ lecture TL » et l'on en déduit la valeur de la dose archéologique.

\section{Détermination de la dose d'irradiation annuelle I}

La dose d'irradiation annuelle rend compte de la radioactivité $\alpha$ et $\beta$ des terres cuites à dater et de la contribution $\gamma$ de l'environnement. Ce dernier est constitué, dans le cas présent, par les murs de la tombe et le sédiment de remplissage. Précisons que lors de l'inhumation, la sépulture étant creuse, les rayonnements $\gamma$ très faiblement absorbés par l'air confiné provenaient de chaque paroi de la chambre funéraire, dont la composition est considérée comme identique à celle du sédiment de remplissage. Afin de déterminer la dose d'irradiation reçue chaque année par les céramiques à dater, différentes techniques ont été utilisées. Sur le terrain, des mesures directes de la radioactivité ont été réalisées par gammamétrie in situ, grâce à un gammamètre Studsvik 2414A à scintillateur plastique NE102A (Schvoerer et al. 1994). Au laboratoire, des analyses radiochimiques des échantillons à dater et des matériaux environnants, par spectrométrie gamma à bas bruit de fond (Guibert \& Schvoerer 1991), ont permis d'accéder aux doses d'irradiation annuelles internes $(\alpha$ et $\beta)$ et externes $(\gamma)$. Les conversions teneur en radioéléments/dose annuelle ont été obtenues à l'aide des tables de Nambi et Aitken (1986). La contribution des rayonnements cosmiques a été évaluée à partir de la profondeur d'enfouissement des céramiques à dater (Prescott \& Hutton 1994). La dose externe a finalement été calculée en combinant les mesures de terrain et les analyses de laboratoire. 
RÉSULTATS EXPÉRIMENTAUX

\section{Mesure des doses d'irradiation archéologiques}

\section{Conditions expérimentales de mesure de la TL}

L'enregistrement des signaux de TL a été réalisé lors d'un chauffage des cristaux à dater en atmosphère d'azote, depuis la température ambiante jusqu'à $500^{\circ} \mathrm{C}$, à la vitesse de $4^{\circ} \mathrm{C} /$ seconde. Un palier est effectué à $245^{\circ} \mathrm{C}$ pendant 2 minutes, de façon à éliminer les composantes instables de la thermoluminescence susceptibles d'entraver l'exploitation des signaux pour la datation. La détection des émissions de TL a été assurée par un tube photomultiplicateur, de type EMI 9813/QKA, couplé à des filtres optiques sélectionnant les composantes spectrales comprises entre 350 et $500 \mathrm{~nm}$ (Schott BG12), qui correspondent aux émissions des silicates - quartz et feldspaths potassiques - contenus dans les terres cuites. La mise en place d'un filtre MTO Ta2 a permis d'atténuer le rayonnement du corps noir dans les longueurs d'onde infrarouge et rouge.

\section{Stabilité thermique des signaux de TL}

L'étude de la stabilité dans le temps des signaux de TL exploités pour la datation n'a pas révélé de perte significative d'intensité à la suite d'une irradiation récente, ce qui aurait pu traduire un phénomène de fading anormal (Visocekas 1985; Sanderson 1988). En conséquence, la stabilité thermique du pic de TL à $350^{\circ} \mathrm{C}$ utilisé pour déterminer les doses d'irradiation archéologiques Qnat». et Qnat $\beta$ paraît assurée.

\section{Résultats obtenus}

Les enregistrements de TL obtenus pour les cinq tessons étudiés présentent une bonne reproductibilité. En exemple, sont présentées les courbes de première lecture de l'échantillon BDX 5130 (Figure 3a). Le domaine d'étude a été choisi entre $330^{\circ} \mathrm{C}$ et $400^{\circ} \mathrm{C}$ à l'aide du test du plateau (Figure $3 \mathrm{~b}$ ). L'intégration des courbes de TL dans ce domaine conduit à la détermination de la dose archéologique par approximation polynomiale, comme illustré sur la Figure 4.

Les résultats de l'étude de TL sont récapitulés dans le Tableau 2.

\section{Mesure des doses d'irradiation annuelles}

Afin de déterminer avec une grande fiabilité les doses d'irradiation annuelles reçues par les céramiques provenant de la tombe du Prêtre (Tableau 2), nous avons procédé à une étude globale de la radioactivité du site de Sipán et de son évolution au cours du temps. Cela supposait la prise en compte de l'ensemble des informations recueillies sur le terrain, dans les différents contextes funéraires à dater et, au laboratoire, sur les prélèvements de céramiques et de sédiments associés à chaque sépulture. 


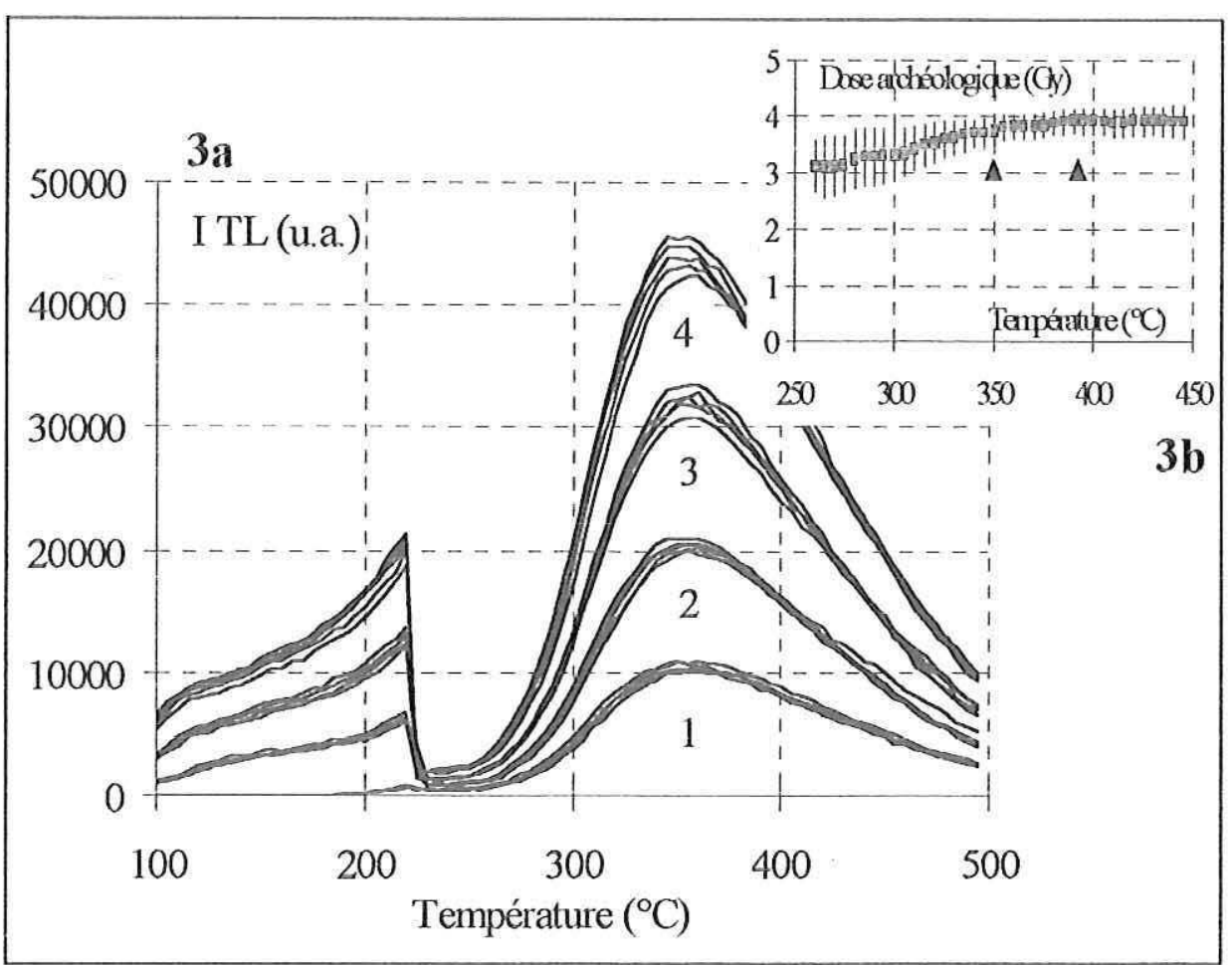

FIG. 3 - BDX 5130, tombe du Prêtre, Sipán: ensemble des courbes de thermoluminescence dites de " première lecture » (3a). Dans l'ordre d'intensité croissante, on trouve : les courbes de TL naturelle (1), les courbes de TL naturelle + 4.3Gy (2), les TL naturelles + 8.6Gy (3) et les TL naturelles + 12.8Gy (4). Elles sont présentées soustraites de leur bruit de fond. On peut remarquer la très bonne reproductibilité de l'ensemble. Le test du plateau réalisé à partir de cette série de courbes de thermoluminescence, qui représente la variation de la dose archéologique en fonction de la température, montre un domaine constant entre $350^{\circ} \mathrm{C}$ et $390^{\circ} \mathrm{C}$, délimité par les deux flèches (3b).

Composition radiochimique et déséquilibre des chaînes radioactives de l'uranium

L'examen des teneurs en radioéléments mesurées sur les matériaux prélevés à Sipán, céramiques et sédiments, permet de mettre en évidence une relative homogénéité radiochimique de l'ensemble. De plus, il apparaît que les concentrations en uranium données par les éléments dits « tête de chaîne » ont tendance à être supérieures à celles déduites de l'analyse des éléments de « fin de chaîne » (Guibert et al. 1994 et 1997). C'est là la preuve d'un déséquilibre des séries radioactives de l'uranium qui pourrait être une conséquence de la migration de radioéléments sous l'effet de la circulation ou d'une percolation d'eau durant l'enfouissement. La procédure utilisée afin d'identifier l'élément responsable du déséquilibre a reposé sur l'analyse de la variance des rapports $\mathrm{U}\left({ }^{238} \mathrm{U}\right) / \mathrm{Th}$ et $\mathrm{U}\left({ }^{226} \mathrm{Ra}\right) / \mathrm{Th}$, présentée dans le Tableau 3 . Une plus grande variabilité de $U\left({ }^{238} \mathrm{U}\right)$ par rapport à $U\left({ }^{226} \mathrm{Ra}\right)$ est ainsi mise en évidence : elle vaut en effet $1,47.10^{-3}$ pour $U$ contre $0,36.10^{-3}$ pour Ra, après déduction de la variance due aux incertitudes statistiques de mesure. Nous en concluons ainsi qu'à 


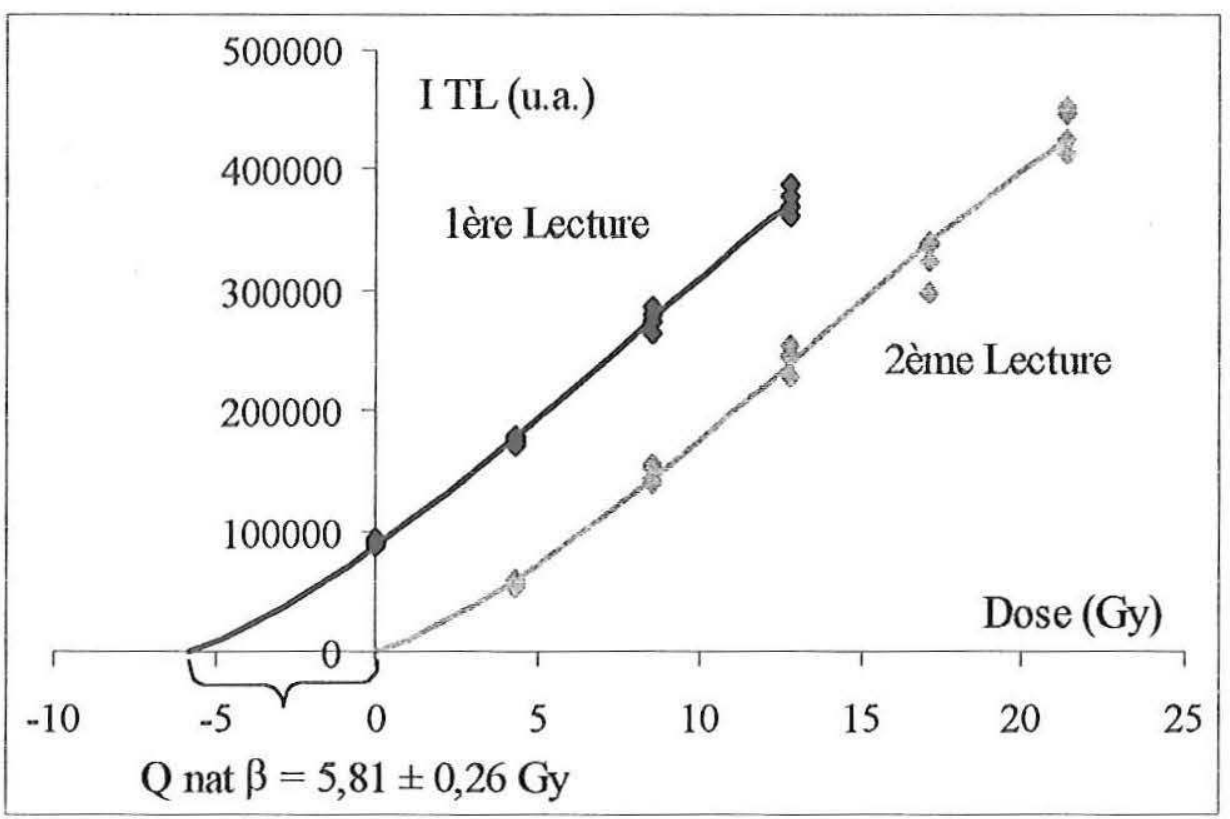

FIg. 4 - BDX 5130, tombe du Prêtre, Sipán : détermination de la dose d'irradiation archéologique. Les points de " première lecture » et de « deuxième lecture » correspondent à l'intégration des courbes de TL dans l'intervalle de température du plateau sélectionné précédemment. La loi de variation de la thermoluminescence en fonction de la dose d'irradiation, déterminée par approximation polynomiale à partir des points du second ensemble, est transposée à la première série d'expériences. L'intersection de cette courbe avec l'axe des doses détermine la dose archéologique ; ici $\mathrm{Q}_{\mathrm{nat} \beta}=5.81 \pm 0.26 \mathrm{~Gy}$.

Sipán, c'est la mobilité de l'uranium qui est le plus probablement à l'origine du déséquilibre observé, bien qu'il soit impossible de déterminer quand cette perturbation a eu lieu. Elle reste néanmoins de faible amplitude. En conséquence, dans le cadre de la datation des céramiques extraites de la tombe du Prêtre, c'est un modèle de variation progressive en élément uranium qui a été considéré pour le calcul des doses annuelles internes (alpha et bêta) et externes (gamma), selon la procédure décrite par ailleurs (Guibert et al. 1997).

\section{Mesures d'humidité}

Les mesures d'humidité effectuées au moment du prélèvement des céramiques montrent des valeurs faibles, voire nulles : les matériaux ont séché sur place, les tombes ayant été fouillées et exposées à la chaleur ambiante longtemps avant la campagne d'échantillonnage en vue de la datation. Dans ces conditions, toutes les teneurs en eau sont sous-estimées et ne peuvent rendre compte des conditions de conservation archéologiques. Ce sont alors les mesures d'eau effectuées à saturation (représentant la quantité maximale d'eau que peut contenir une céramique) qui ont été exploitées afin d'évaluer l'état d'humidité moyen des tessons tout au long de leur enfouissement. Dans le cas présent, il semble que le taux fixé à $50 \%$ de la saturation puisse être 
considéré comme représentatif (cela revient à prendre en compte un $\% \mathrm{H}_{2} \mathrm{O}$ compris entre $2 \%$ et $7 \%$ selon les échantillons, cette variabilité apparente rendant compte des écarts de porosité observés entre les céramiques, dus à des états de cuisson sensiblement différents). L'incertitude associée à cette mesure a été prise à un écart-type, en considérant que toutes les valeurs comprises entre $0 \%$ et $100 \%$ de la saturation étaient équiprobables. Ce sont ces données qui ont été utilisées pour le calcul des doses d'irradiation annuelles internes. En ce qui concerne les sédiments, et pour les mêmes raisons que celles décrites précédemment, il apparaît que les mesures d'humidité effectuées sur la majorité des matériaux sont sous-évaluées. Néanmoins, les échantillons prélevés à l'intérieur des parois, dans les galeries gammamétriques, conservent une teneur en eau comprise entre $3 \%$ et $6 \%$, jugée représentative de l'état « archéologique » naturel. Dans ces conditions, ce résultat peut être étendu aux autres éléments de l'environnement et un pourcentage d'eau égal à $6 \pm 3 \%$ a été pris en compte dans le cadre de la détermination des doses d'irradiation annuelles externes.

\section{Âge-TL des céramiques de la tombe du Prêtre}

Les âges déterminés par TL pour les cinq tessons de céramiques associés à la tombe du Prêtre sont rassemblés dans le Tableau 4.

Ces datations présentent une dispersion statistique (79 ans) voisine de l'incertitude des résultats. Le calcul du « chi 2 », traduisant la normalité de la distribution, révèle néanmoins une légère anomalie (intervalle compris entre 1.06 et 7.78 à $80 \%$ de probabilité, d'après CEA, anonyme, 1978). Les expériences et mesures réalisées sur les cinq échantillons ne permettant pas d'identifier le résultat anormal, nous avons choisi de conserver tous les âges et de raisonner sur leur moyenne arithmétique. La tombe du Prêtre de Sipán est ainsi datée à :

$$
1262 \pm 54 \text { ans, soit } 736 \pm 54 \mathrm{AD}(\text { à } 1 \sigma) \text {. }
$$

\section{Confrontation TL $/{ }^{14} \mathrm{C}$, BILAN ET Discussion}

\section{Un résultat complémentaire de datation par radiocarbone}

La datation par ${ }^{14} \mathrm{C}$ réalisée sur le fragment de poutre (BDX 5138), pour lequel on pouvait garantir l'absence de pollution par des consolidants organiques fréquemment utilisés à Sipán lors des fouilles, donne un résultat en bon accord avec ceux précédemment acquis par TL : $1190 \pm 80 \mathrm{BP}$, soit après calibration [770-960] $\mathrm{AD}$ à $1 \sigma$ et [680-990] AD à $2 \sigma$ (d'après la courbe publiée par Stuiver \& Reimer en 1993 et la prise en compte du fractionnement isotopique).

Apport de ces nouvelles données physiques pour la connaissance de la chronologie du site de Sipán

Grâce au croisement de deux méthodes indépendantes, la TL et le ${ }^{\mathrm{I}}{ }^{\mathrm{C}} \mathrm{C}$, mises en œuvre sur des matériaux de natures différentes, il est désormais possible de placer, avec 


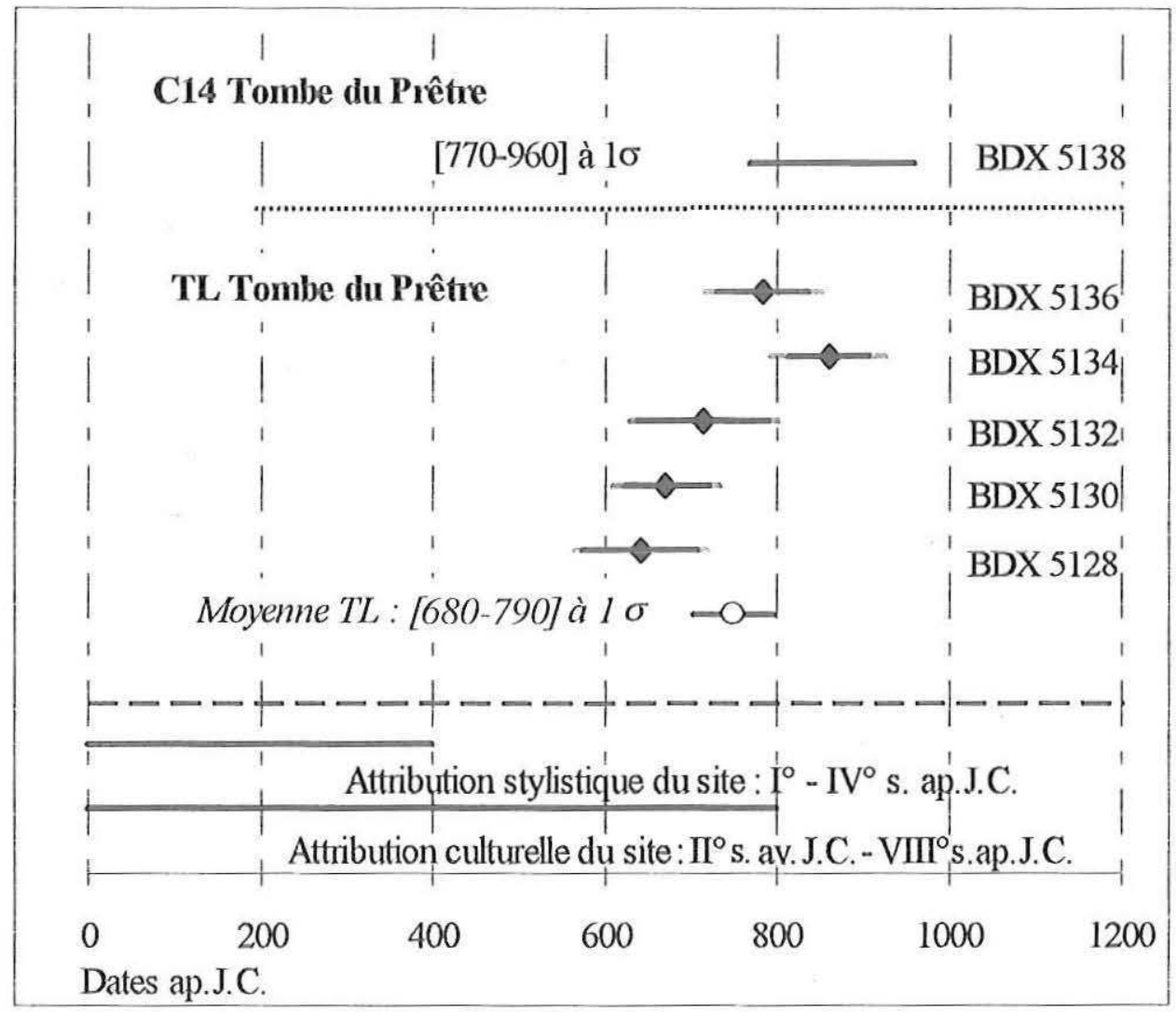

FIG. 5 - Tombe du Prêtre, Sipán : datations obtenues par thermoluminescence sur les céramiques (dates par échantillon et moyenne pondérée) et par ${ }^{14} \mathrm{C}$ sur un fragment de poutre scellant la chambre funéraire. Les deux ensembles de résultats sont en bon accord et permettent de placer la tombe du Prêtre au milieu du $\mathrm{V}^{\mathrm{e}}{ }^{\mathrm{e}}$ siècle de notre ère. Ces données chronologiques physiques divergent de l'attribution initiale de la nécropole à la période $\mathrm{I}^{\mathrm{e}}-\mathrm{IV}^{\mathrm{e}}$ siècle de notre ère.

un bon degré de confiance, l'inhumation du Prêtre dans le courant du viII ${ }^{\mathrm{e}}$ siècle de notre ère. Ce résultat peut être comparé (Figure 5) aux données chronologiques précédemment connues pour la nécropole de Sipán reposant sur des considérations typologiques, stylistiques et architecturales. Il diffère significativement du calage de la nécropole entre les $\mathrm{I}^{\mathrm{er}}$ et IV ${ }^{\mathrm{e}}$ siècles de notre ère et, a fortiori, de la première datation ${ }^{14} \mathrm{C}$ relative à la tombe du Seigneur $(260 \pm 90 \mathrm{AD})$. D'un point de vue plus large, les datations physiques relatives à la tombe du Prêtre concordent toujours avec une attribution du site à la civilisation mochica qui, si l'on se réfère aux chronologies générales des cultures pré-hispaniques du Pérou, s'éteint dans le courant du VIII ${ }^{\mathrm{e}}$ siècle de notre ère.

Les recherches à caractère chronologique engagées sur la nécropole princière de Sipán mettent d'ores et déjà en lumière les insuffǐsances du système typochronologique habituellement utilisé et apportent un éclairage tout à fait nouveau sur 
la place de ce site dans le développement temporel de la culture mochica. Elles seront poursuivies par la datation de céramiques provenant d'autres tombes de la plateforme funéraire, afin de parvenir à un calage chronologique fiable et rigoureux de ce site majeur pour l'histoire pré-hispanique du Pérou.

TABleau 1 - Tombe du Prêtre, Sipán : localisation et présentation succincte des différents matériaux prélevés pour la datation par TL.

\begin{tabular}{|c|c|c|c|c|}
\hline $\begin{array}{l}\text { Référence } \\
\text { Céramique }\end{array}$ & $\begin{array}{c}\text { Point de } \\
\text { prélèvement }\end{array}$ & Typologie & $\begin{array}{l}\text { Dimensions } \\
(\mathrm{mm}) \\
\mathrm{L} \times \mathrm{I} \times \mathrm{e}\left({ }^{1}\right)\end{array}$ & $\begin{array}{l}\text { Sédiment } \\
\text { associé }\end{array}$ \\
\hline BDX 5128 & $\begin{array}{l}\text { Hornacina } 1 \\
\text { angle S-E }\end{array}$ & $\begin{array}{l}\text { Olla ou jarre } \\
\text { fragments de } \\
\text { couvercle }\end{array}$ & $\begin{array}{l}\text { Ensemble: } \\
99 \times 62 \times 6\end{array}$ & $\begin{array}{l}\text { BDX } 5129\left({ }^{2}\right) \\
\text { BDX } 5262\left({ }^{3}\right)\end{array}$ \\
\hline BDX 5130 & $\begin{array}{l}\text { Hornacina } 1 \\
\text { angle S-E }\end{array}$ & $\begin{array}{c}\text { Olla } \\
\text { fragments } \\
\text { jointifs de } \\
\text { lèvre et de } \\
\text { panse }\end{array}$ & $\begin{array}{l}\text { Ensemble : } \\
132 \times 90 \times 4\end{array}$ & $\begin{array}{l}\text { BDX } 5131\left({ }^{2}\right) \\
\text { BDX } 5262\left({ }^{3}\right)\end{array}$ \\
\hline BDX 5132 & $\begin{array}{c}\text { Hornacina } 2 \\
\text { angle N-E }\end{array}$ & $\begin{array}{l}\text { Petit vase bas } \\
\text { globulaire } \\
\text { fragments } \\
\text { jointifs de col }\end{array}$ & $\begin{array}{l}\text { Ensemble: } \\
86 \times 64 \times 5\end{array}$ & BDX $5133\left({ }^{2}\right)$ \\
\hline BDX 5134 & $\begin{array}{c}\text { Hornacina } 3 \\
\text { angle } \mathrm{N}-\mathrm{O}\end{array}$ & $\begin{array}{c}\text { Olla } \\
\text { fragments } \\
\text { jointifs de col }\end{array}$ & $\begin{array}{c}\text { Ensemble : } \\
132 \times 78 \times 7\end{array}$ & BDX $5135\left(^{2}\right)$ \\
\hline BDX 5136 & $\begin{array}{c}\text { Hornacina } 4 \\
\text { angle S-O }\end{array}$ & $\begin{array}{c}\text { Olla } \\
\text { fragments, } \\
\text { dont deux } \\
\text { jointifs, de } \\
\text { lèvre et de } \\
\text { panse }\end{array}$ & $\begin{array}{c}\text { Fragments: } \\
1 \cdot 76 \times 69 \times 4 \\
2 \cdot 72 \times 37 \times 4 \\
3 \cdot 65 \times 56 \times 4 \\
4 \cdot 50 \times 43 \times 4\end{array}$ & $\begin{array}{l}\text { BDX } 5137\left(^{2}\right) \\
\text { BDX } 5263\left(^{3}\right)\end{array}$ \\
\hline
\end{tabular}

( ') $\mathrm{L}=$ longueur, $\mathrm{l}=$ largeur, $\mathrm{e}=$ épaisseur.

$\left({ }^{2}\right)$ au contact ou à proximité du tesson à dater.

$\left({ }^{3}\right)$ dans le trou de gammamétrie, cavité horizontale creusée pour recevoir la tête de la sonde de mesure de la radioactivité « $\gamma+\operatorname{cosmique~»,~à~proximité~immédiate~du~point~de~prélèvement~du~tesson~à~dater.~}$ 
Tableau 2 - Tombe du Prêtre, Sipán : datation par TL des céramiques (nd : non déterminé).

\begin{tabular}{|c|c|c|c|c|c|}
\hline $\begin{array}{l}\text { Céramiques } \\
\text { Tombe du } \\
\text { Prêtre }\end{array}$ & BDX 5128 & BDX 5130 & BDX 5132 & BDX 5134 & BDX 5136 \\
\hline Qnat $\beta$ (Gy) & $6.00 \pm 0.37$ & $5.81 \pm 0.26$ & $5.78 \pm 0.42$ & $5.76 \pm 0.28$ & $6.05 \pm 0.33$ \\
\hline $\mathrm{k}\left(\mathrm{Q}_{\text {natp }} / \mathrm{Q}_{\text {nata }}\right)$ & $0.070 \pm 0.005$ & $0.083 \pm 0.006$ & $0.100 \pm 0.012$ & $0.108 \pm 0.009$ & $0.098 \pm 0.009$ \\
\hline \multicolumn{6}{|l|}{$\begin{array}{c}\text { Dose annuelle } \\
\text { interne } \\
\text { (mGy/an) }\end{array}$} \\
\hline $\mathrm{kI} \alpha$ & $1.01 \pm 0.10$ & $1.06 \pm 0.10$ & $1.32 \pm 0.18$ & $1.56 \pm 0.17$ & $1.55 \pm 0.17$ \\
\hline I $\beta$ & $1.93 \pm 0.05$ & $1.84 \pm 0.06$ & $1.89 \pm 0.06$ & $2.14 \pm 0.10$ & $2.13 \pm 0.08$ \\
\hline I interne & $2.94 \pm 0.11$ & $2.90 \pm 0.12$ & $3.21 \pm 0.19$ & $3.70 \pm 0.20$ & $3.68 \pm 0.19$ \\
\hline \multicolumn{6}{|l|}{$\begin{array}{c}\text { Dose annuelle } \\
\text { externe } \\
\text { (mGy/an) }\end{array}$} \\
\hline $\mathrm{I} \gamma$ & $1.38 \pm 0.04$ & $1.38 \pm 0.04$ & $1.18 \pm 0.04$ & $1.25 \pm 0.04$ & $1.18 \pm 0.04$ \\
\hline I cosmiques & $0.12 \pm 0.03$ & $0.12 \pm 0.03$ & $0.12 \pm 0.03$ & $0.12 \pm 0.03$ & $0.12 \pm 0.03$ \\
\hline $\mathrm{I}\left(\mathrm{I} \gamma+\mathrm{I}_{\mathrm{cos}}\right)$ & $1.50 \pm 0.03$ & $1.50 \pm 0.03$ & $1.30 \pm 0.03$ & $1.37 \pm 0.05$ & $1.30 \pm 0.03$ \\
\hline I $\gamma$ métrie in situ & $1.47 \pm 0.07$ & $1.47 \pm 0.07$ & nd & nd & $1.32 \pm 0.07$ \\
\hline $\begin{array}{c}\text { Moyenne I } \\
\text { externe }\end{array}$ & $1.49 \pm 0.03$ & $1.49 \pm 0.03$ & $1.30 \pm 0.03$ & $1.37 \pm 0.05$ & $1.31 \pm 0.03$ \\
\hline $\begin{array}{l}\text { Dose annuelle } \\
\text { totale (mGy/an) }\end{array}$ & $4.43 \pm 0.12$ & $4.39 \pm 0.12$ & $4.51 \pm 0.20$ & $5.07 \pm 0.21$ & $4.99 \pm 0.19$ \\
\hline
\end{tabular}

TABLEAU 3 - Céramiques de Sipán : étude des rapports U $\left({ }^{238} \mathrm{U}\right) / \mathrm{Th}$ et $\mathrm{U}\left({ }^{226} \mathrm{Ra}\right) / \mathrm{Th}$. Les résultats montrent que c'est le rapport $U\left({ }^{238} \mathrm{U}\right) / \mathrm{Th}$ qui présente la variance la plus élevée après déduction de la contribution des incertitudes de mesure: $1,47.10^{-3}$ contre $0,36 \cdot 10^{-3}$ pour $\mathrm{U}\left({ }^{226} \mathrm{Ra}\right) / \mathrm{Th}$. On en déduit donc que, globalement, les matériaux ont subi une modification de leur composition en uranium.

\begin{tabular}{|l|r|r|}
\cline { 2 - 3 } \multicolumn{1}{c|}{} & $\begin{array}{r}\text { Rapport } \\
\mathbf{U}\left({ }^{238} \mathbf{U}\right) / \mathbf{T h}\end{array}$ & $\begin{array}{c}\text { Rapport } \\
\mathbf{U}\left({ }^{226} \mathbf{R a}\right) / \mathbf{T h}\end{array}$ \\
\hline Moyenne & 0,322 & 0,276 \\
\hline Ecart-type & 0,052 & 0,020 \\
\hline Ecart-type relatif (\%) & 11,9 & 6,8 \\
\hline Variance (V) & $2,67.10^{-3}$ & $0,39.10^{-3}$ \\
\hline Variance due aux analyses $\left(\mathrm{V}_{\text {an }}\right)$ & $1,20.10^{-3}$ & $3,6.10^{-5}$ \\
\hline Variance nette $\left(\mathrm{V}-\mathrm{V}_{\text {an }}\right)$ & $\mathbf{1 , 4 7 . 1 0 ^ { - 3 }}$ & $\mathbf{0 , 3 6 . 1 0 ^ { - 3 }}$ \\
\hline
\end{tabular}


TABleau 4 - Tombe du Prêtre, Sipán : datation par TL des cinq céramiques prélevées dans la sépulture. Cet ensemble donne d'ores et déjà une nouvelle idée de la chronologie du site.

\begin{tabular}{|c|c|c|c|c|}
\hline $\begin{array}{c}\text { Céramiques } \\
\text { Tombe du Prêtre }\end{array}$ & $\begin{array}{c}\text { Age-TL (ans) } \\
\text { Incertitude } \\
\text { totale (à 1 } \sigma)\end{array}$ & $\begin{array}{c}\text { Incertitude } \\
\text { statistique }\end{array}$ & $\begin{array}{c}\text { Incertitude } \\
\text { systématique }\end{array}$ & $\begin{array}{c}\text { Date } \\
\text { (AD) }\end{array}$ \\
\hline BDX 5128 & $1355 \pm 76$ & 67 & 36 & $642 \pm 76$ \\
\hline BDX 5130 & $1325 \pm 62$ & 50 & 36 & $672 \pm 62$ \\
\hline BDX 5132 & $1282 \pm 87$ & 77 & 39 & $715 \pm 87$ \\
\hline BDX 5134 & $1136 \pm 66$ & 47 & 47 & $861 \pm 66$ \\
\hline BDX 5136 & $1213 \pm 68$ & 54 & 41 & $784 \pm 68$ \\
\hline
\end{tabular}

\section{RÉFÉRENCES BIBLIOGRAPHIQUES}

Aitken, M. J., 1985. - Thermoluminescence dating, Academic Press (England), 359 p.

Aitken, M. J., P. A. Clarck \& C. F. Gaffney, 1985. - « Beta and gamma gradients », Nuclear Tracks, vol. 10, $\mathrm{n}^{\circ} 4-6$, pp. 647-653.

Alcina Franch, J., 1996. - L'art précolombien, Éd. Citadelles et Mazenod, pp. 99-205 et p. 537.

AlvA, W., 1988. - « Discovering the new word's richest unlooked Tomb », National Geographic, tome 174, vol. 4, pp. 510-549, Washington, DC.

—, 1990. — « New tomb of royal splendor », National Geographic, tome 177, vol. 6, pp. 2-15, Washington, DC.

—, 1994. - Sipán, Colección Cultura y Artes del Perú. Edición dirigida por José Antonio de Lavalle, Cervecería Backus y Johnston SA.

Alva, W. \& C. B. Donnan, 1993. - Royal tombs of Sipán, Fowler Museum of Natural History, Los Angeles, UCLA.

Arsenault, D., 1995. - «Balance de los estudios moche (Mochicas), 1970-1994 ", Revista Andina, vol. 13, part. 1, pp. 237-270, et part. 2, pp. 443-480.

BAwden, G., 1994. - « La paradoja estructural : la cultura Moche como ideología política », in : S. Uceda y E. Mujica (eds.), Moche : propuestas y perspectivas. Actas del primer coloquio sobre la cultura moche, Universidad de Trujillo, pp. 389-412, IFEA y APFCS, Lima.

—, 1996. - The Moche, Coll. The peoples of America, Blackwell Publishers, 375 p.

CEA, anonyme, 1978. - Statistique appliquée à l'exploitation des mesures, tome 1, Edition Masson, $148 \mathrm{p}$.

Canziani, A. J., S. Uceda y B. E. MujicA, 1994. - « Perspectivas de los estudios sobre la cultura Moche ", in : S. Uceda y E. Mujica (eds), Moche, op. cit., pp. 495-500.

Collin Delavaud, C., 1984. - Las regiones costeñas del Perú septentrional, Pontificia Universidad Católica del Perú, Fondo Editorial. 
Curatola, M. y F. Silva-Santisteban (eds.), 1995. - Historia y cultura del Perú, Universidad de Lima, Museo de la Nación, Banco de crédito del Perú, 450 p.

Donnan, C. B., 1965. - « Moche ceramic technology », Nawpa Pacha, vol. 3, pp. 115-134, Institute of Andean Studies, Berkeley.

-, 1976. - Moche Art and Iconography, UCLA Latin American Center Publications, University of California, Los Angeles.

Donnan, C. B. \& D. Mc Clelland, 1979. - The Burial Theme in Moche Iconography, Studies in Pre-Columbian Art and Archaeology, vol. 21, Dumbarton Oaks, Washington, DC.

Donnan, C. B. \& L. J. Castillo, 1992. — «Finding the tomb of a Moche Priestess ", Archaeo$\log y$, vol. 45 , part. 6 , pp. 38-42.

GUiBERT, P. \& M. SCHVOERER, 1991. — « TL-dating : low background gamma spectrometry as a tool for the determination of the annual dose », Nuclear Tracks and Radiation Measurements, vol. 18, $\mathrm{n}^{\circ} 1 / 2$, pp. 231-238.

Guibert, P., M. Schyoerer, M. P. Etcheverry, B. Szepertyski \& C. Ney, 1994. - « IX ${ }^{\text {th }}$ Millenium BC ceramics from Niger : detection of U-series desequilibrium and TL-dating ", Quaternary Geochronology, vol. 13, pp. 555-561.

Guibert, P., E. Vartanian, F. Bechtel \& M. Schvoerer, 1996. - « Non linear approach of TL response to dose : polynomial approximation ", Ancient-TL, vol. 14, n 2, pp. 7-14.

Guibert, P., F. Bechtel \& M. Schvoerer, 1997. - «Déséquilibre des séries de l'uranium, implications sur la dose annuelle en datation par thermoluminescence : une étude à la Grotte XVI, Cénac et Julien, Dordogne (France) ", Quaternaire, vol. 8, pp. 377-389.

Guibert, P., C. Ney, F. Bechtel, M. Schvoerer \& P. Araguas, 1998. - « Datation par thermoluminescence d'éléments architecturaux en terre cuite de la Seo del Salvador, église cathédrale de Saragosse, Espagne », Revue d'Archéométrie, vol. 22, pp. 125-135.

Larco Hoyle, R., 1948. - Cronología arqueológica del norte del Perú, Biblioteca del Museo de Arqueología Rafael Larco Herrera, Sociedad Geográfica Americana, Hacienda Chiclín, Buenos Aires.

LÉvine, D., 2001. — « Des "vérités établies" aux évidences actuelles, la quête d'une chronologie », Colloque "Datation », $\mathrm{xxI}^{\mathrm{e}}$ Rencontres Internationales d'Archéologie et d'Histoire d'Antibes, 19-21 octobre 2000, pp. 433-437.

Makowski Hanula, K., 1995. - « Las grandes culturas de la costa norte », in : M. Curatola y F. Silva-Santisteban (eds.), Historia y cultura del Perú, Universidad de Lima, Museo de la Nación, Banco de crédito del Perú, pp. 101-145.

NAmbi, K. S. V. \& M. J. Aitken, 1986. - « Annual dose conversion factors for TL and ESR dating ", Archaeometry, vol. 28, part 2, pp. 202-205.

Prescott, J.-R. \& J. T. Hutton, 1994. — « Cosmic ray contributions to dose rates for luminescence and ESR dating : large depths and long-term time variations ", Radiation Measurements, vol. 23, $\mathrm{n}^{\circ}$ 2/3, pp. 497-500.

SAnderson, D. C. W., 1988. - « Fading of TL in feldspars : characteristics and corrections », Nuclear Tracks and Radiation Measurements, vol. 14, n 1/2, pp. 155-161.

Schuster, A. M. H., 1992. - «Inside the royal tombs of the Moche », Archaeology, vol. 45, $\mathrm{n}^{\circ} 6, \mathrm{pp} .30-36$.

Schvolrer, M., P. Guibert \& C. Ney, 1994. - «Chronologie par thermoluminescence de cultures pré-hispaniques de l'Ouest mexicain : étude de céramiques provenant de deux sondages (puits 1 et 13) du site de Coamiles "), Revue d'Archéométrie, vol. 18, pp. 23-42. 
Shimada, I., 1994. - " Los modelos de la organización sociopolítica de la cultura Moche », in : S. Uceda y E. Mujica (eds.), Moche : propuestas y perspectiva. Actas del primer coloquio sobre la cultura Moche, pp. 359-387, Universidad de Trujillo, IFEA y APFCS, Lima.

Stuiver, M. \& P. J. Reimer, 1993. - « Calibration 1993 », Radiocarbon, vol. 35, nº 1, pp. 215230.

UCEDA, S., 2000. — « La cronología Moche, problemática y alternativas actuales », Séminaire PACT-Trujillo, Coopération Europe-Amérique latine pour la préservation et la valorisation de l'héritage culturel, 29 janvier- ${ }^{\text {er }}$ février 2000 , communication orale.

UCEDA, S. y B. E. Mujica (eds.), 1994. - Moche: propuestas y perspectivas. Actas del primer coloquio sobre la cultura Moche, Universidad de Trujillo, IFEA y APFCS, Lima, 549 p.

VisoceKas, R., 1985. - « Tunneling radiative recombination in labradorite : its association with anomalous fading ", $P A C T$, vol. 3, pp. 258-265.

WAtanabe, L. K., 1995. - «Una version sobre los mochicas, 200 años A.C.-750 años D.C. ", in : Culturas preincas del Perú, Coll. Nuestra Historia, Fondo Editorial de Cofide, pp. 193258. 